\title{
The effect of an educational intervention in improving hypertension-related adherence to medication and health-related quality of life
}

\author{
Hira Iqbal ${ }^{1, *}$, Hafiz Rashid Hussain ${ }^{2}$, Farwa Zara ${ }^{3}$, Mehrunnisa ${ }^{4}$ \\ ${ }^{1,3,4}$ Student, ${ }^{2}$ Assistant Professor, College of Pharmacy, University of Sargodha, Sargodha, Punjab, Pakistan \\ *Corresponding Author: \\ Email: bluediamond7867@gmail.com
}

\begin{abstract}
The objective of this study is to evaluate the effect of educational intervention in improving hypertension-related adherence to medication and health-related quality of life. This study includes the effects of pharmacist interventions on cardiovascular problems, economics, medication issues, medicine adherence, self-prescription, hospitalized patients and discharged patients. The review study shows that pharmacist and physician co management in order to control blood pressure is significant at decreasing blood pressure and rate of blood pressure control is improved. The pharmacist can also improve medication adherence, quality of life and patient knowledge about diseases and medication and give satisfaction to patient. In addition, the use of drugs is reduced. This study emphasizes that intervention of pharmacist in medication management or therapy is health effective. As a member of health care team pharmacist can increase the number of health outcomes. Hypertension is an important risk factor contributes to present epidemic cardiovascular problems with the pharmacist intervention in controlling of blood pressure its risk is going to decrease.
\end{abstract}

Keywords: Hypertension, Role of pharmacist, Essential hypertension, Cardio-vascular problems, Adherence.

\section{Introduction}

High blood pressure is defined asa consistently elevated blood pressure exceeding 140/90 mm Hg. In essential hypertension $(95 \%$ of people with hypertension), no specific cause is found. High blood pressure is called "the silent killer" because it often causes no symptoms for many years, even decades, until it damages certain criticalorgans. ${ }^{1}$ It is most usual cause of many cardiovascular problems all over the world. $^{2,3}$

Actually blood pressure is the combination of two measurements:

a. Systolic blood pressure

b. Diastolic blood pressure

Normal BP: normal blood pressure value is $120 / 80$ mmHg. ${ }^{4,5}$

High blood pressure: if $\mathrm{BP}$ value is greater than 140/90mmHg. ${ }^{6}$

Primary HTN and essential HTN are the types of hypertension. (22)Essential hypertension is most common form of hypertension and present in $95 \%$ of patient suffering with hypertension. ${ }^{7}$ High blood pressure diagnosed in one of every three American individuals. An evaluation has been made from national health and nutrition examination survey that control in high blood pressure increased from twenty seven percent to fifty percentup to $2008 .^{8}$ Globally, high blood pressure is estimated to cause 7.1 million deaths, about $13 \%$ of the total. About $62 \%$ of cerebrovascular heart disease is attributable to suboptimal BP (systolic $>115 \mathrm{~mm} \mathrm{Hg})^{8}$

The risk of heart problems and stoke mortality may be decrease from $25 \%$ to $30 \%$ for every5.8 $\mathrm{mmHg}$ decrease in diastolic blood pressure due to pharmacist intervention. ${ }^{9}$ Team-based care is the strategy that has had the greatest effect on improving blood pressure (BP). The purpose of this systematic review was to determine the potency of interventions for BP involving nurses or pharmacists. ${ }^{10}$ In 1998, according to national health survey in Pakistan, prevalence of hypertension reported that it affects $17 \%$ of overall population. ${ }^{11}$ It was estimated that that $26 \%$ of adult people have hypertension problem in year $2000 .{ }^{12}$

Symptoms of Hypertension: High blood pressure is actually a silent problem to an individual. Most of the individual will not have any of the below mentioned symptoms. It sometime takes many years or decades to reach sever level enough that some symptoms become appear. Even at that time appeared symptoms may associated to some other health problem.

Symptoms are:

1. Feeling of headache.

2. Pain in chest.

3. Changes in vision.

4. Presence of blood in urine.

5. Bleeding from nose.

6. Problem in breathing (SOB).

7. Flushing and dizziness.

All of these symptoms don't appear in all patients of hypertension but wait until these symptoms appear might be dangerous.

Role of Pharmacist: WHO stated that the effectiveness of adherence due to intervention has increased and it may have great impact on population health than any kind of improvement in medical treatment. 
Study have demonstrated that high blood pressure remains ill managed worldwide, due to lack of adherence to medication require for BP lowering is main factor. Hypertensive patient may fail to intake their required medication because of ineffective symptoms in daily routine, long term therapy, medication adverse effect, lack of knowledge about management and medication cost. ${ }^{5}$ When individuals are prescribed for self-administration of medicines they take about half of their doses advised by physician. Some patients stop medication in the few weeks without informing their physician. Pharmacist is the person who is logical choice and helpful assets in hypertension management program to improve condition of patients, works alone or in team based care. $^{13}$ Physician/pharmacist collaboration achieved significantly better mean BP values and overall BP control rates, primarily by intensification of medication therapy and improving patient adherence. A lot of studies show that pharmacist intervention produces a reduction in patients' blood pressure. ${ }^{14,15}$ In this rural community, a pharmaceutical care program was associated with significant improvements in blood pressure control in hypertensive patients. ${ }^{16}$

A first team based care study was published in 1973. Patients of hypertension were randomized to an intervention group or traditional services of pharmacy. The pharmacist present in community pharmacy evaluated patients suffering with hypertension problem in urban health center present in Detroit and started work closely with physicians which were two in number other than health center included visiting clinic to review recommendations made by pharmacist and medical records with a purpose to change in therapy of patient. Intervention group patients were seen every month for approximately five months in one of the community pharmacies by pharmacist. Blood pressure of patients was measured by physician in physician office and blood pressure deteriorated from 163/93 to $166 / 101$ in control group during the period of 5 months. However improvement in blood pressure in intervention group was from $157 / 99$ to $146 / 90 \mathrm{mmhg}$ If once intervention of team care was discontinued control of blood pressure deteriorated in intervention group. ${ }^{17}$ Recent publications study on collaboration of pharmacist and physician in office of medical community, there was adjusted change in blood pressure(systolic) of $-12 \mathrm{mmHg}$ during six months along with improvement in the control of blood pressure $(34 \%) .{ }^{18}$ The pharmacist intervention in 39 randomized control trial which include 14224 patients. Studies conducted in different countries. Most of them in United States and Canada, 5 in Europe and about 10 in Asia Australia, south America. Most of studies $(n=23)$ done by the intervention of pharmacist in community setup. Inn=16 studies the physician, nurses and pharmacist worked in team base care. Due to pharmacist intervention reduction in systolic and diastolic $\mathrm{BP}$ of $-7.6 \mathrm{mmHg}$ and $-3.9 \mathrm{mmh}$ as compared to usual care. This great reduction occurred due to patient education, recommendations to physician and due to management of medication by pharmacist.

Effect on Cardiovascular Problem: Six studies done on hypertensive patients along with cardiovascular issues. Significant reduction in blood pressure seen due to interventions in patients suffering with cardiovascular diseases associated with co morbidity. Reduction in Systolic blood pressure was noted $5.3 \mathrm{mmHg}$ in 578 individuals having cardiovascular associated co- morbidity vs. 580 of control subjects. Reduction in diastolic BP was $1.9 \mathrm{mmHg}$ in 578 individuals having cardiovascular associated comorbidity vs. 590 of control subjects. ${ }^{19}$

Effect on Economics: Due to economic burden patient fill fewer prescriptions, break the tablets or miss the doses, all these things put them on the risk of adverse outcome. ${ }^{20}$

The Pharmacist works like an advocate for their patients in acquisition of drugs and should help patients of getting medication if individuals are unable to pay the expense of their medicines. Pharmacist guide the patient to get help from social assistance program or social workers, can form account for patient to pay bill in installments. All things depend on the pharmacist and patient trust relationship and Laws. ${ }^{21} 20$ patients results were reported by Morse and colleagues who suffered from resistant hypertension, given them pharmaceutical care. Mean BP before study was noticed $178 / 112 \mathrm{mmHg}$ and average of annual expense of drugs were \$325. After the pharmacist intervention for 5 to 8 months mean BP changed to $137 / 91 \mathrm{mmHg}$ and average of annual expense of drugs was decreased to $\$ 143$.

Effect on Education: The pharmacist has enough knowledge to dispel previous myths and other misinformation of patient that he acquired from social media such as TV, internet, unauthentic websites etc so that patient feels easy to complete the pharmacotherapy. The pharmacist educate the patient, so patient will be able to understand in which way store and carry their medicines without degradation of medicinal products. ${ }^{21}$ Pharmacist educate patient about diseases, counsel about the importance of medication adherence, and give him instructions in oral and written form for selfmonitoring. Prescribed bottles of medicines were labeled with attractive colors and also represent the direction of taking medicine and important precaution showed by images for patient comfort.

Effect on Medication Issue: Joint national committee $(\mathrm{JNC}-\mathrm{VI}) 6^{\text {th }}$ report suggested that encourage pharmacists to monitor patient's medication, to give 
them information about the side effects and drug interactions. ${ }^{22}$

In 5 studies pharmacist noted problems related to drugs. In these studies 822 problems were mentioned in 337 patients in intervention group but no such problem seen in 132 patients present in control group. Types of medicine related problems were including:

a. Prescribing of irrelevant medicine,

b. patients were not getting required effects

c. And experienced side effects from prescribed antihypertensive medication. In intervention groups which include 240 patients in 5 studies, pharmacist resolve 205 of 539 issues by giving information to prescriber and patient. ${ }^{19}$

Effect on Medicine Adherence: Adherence may be defined as the extent to which a patient's behavior (in terms of taking medication, following a diet, modifying habits, or attending clinics) coincides with medical or health advice. ${ }^{23}$ Medication adherence is important modifiable factor that affects the treatment outcome. One study shows that non-adherence is the cause of uncontrolled BP and for chronic diseases non adherence leads to adverse therapeutic outcomes and increase the cost of health care. Adherence of medication is maintained only in 50\% of developed countries for chronic diseases. Review of 15 studies was conducted on hypertensive patients for medicine adherence and control of BP with pharmacist interventions. These interventions include management of medicine, education of patient, reminders to take medication, increase contacts, and clinical pharmacist visits. Significant improvements occur in $88 \%$ studies, but adherence increase in only $44 \%$ of patients. All this improvement was made because of medication adjustment done by pharmacist. ${ }^{3,25}$

One study (older and classic) and 3 recent studies result shows that when community pharmacist serve patient and educate them, monitor their BP, manage drug therapy then BP control seen improved in patients. $\mathrm{BP}$ control seen in two of these studies was improved as they are measured by physicians. In addition to this, two studies results shows improvement occur in quality of life in those patients which were under pharmacist care for 5 to6 months. So community pharmacists form a link between physician and patients.

In 1977, pilot projects were established by California assembly bill 717, in these projects pharmacists received some training to prescribe medicine under supervising physician. 326 patients of hypertension were studied by McGhanet al in pilot study. A panel of some experts who evaluated the performance of two of clinical pharmacists and 3 physicians. Pharmacists rated higher for their selection of suitable and most effective medicine therapy as compared with the physicians. Moreover, about $97 \%$ patients under pharmacist management had controlled their blood pressure as compared with the patients under physician management, in which only $77 \%$ patients had controlled BP. ${ }^{22}$

Self Prescription: Pharmacists can prescribe medication but this all depends on region. Alberta the Canadian province has been first one that allows pharmacists to write prescriptions independently. In this province the patients who get prescriptions on antihypertensive therapy shows a unique outcomes. Now in Alberta pharmacists can apply for prescribing authorization additionally, this allows pharmacist to prescribe the patients for self-limiting conditions, to make a change in the prescription written by some other prescriber, and pharmacist can manage drug therapy which also involve independent starting of medication therapy. Prescribing by pharmacist is recent innovation. ${ }^{18}$

Effect on Hospitalized Patient: Clinical pharmacist plays an important role for the hospitalized patients with more emphasis on patient interaction and collaboration care. A report presented by two institute of medicine that, pharmacists are important for safe use of drugs. Pharmacist participation in daily rounds in hospital increases medicine safety, most essential of all that is physician-pharmacist collaboration. Recent survey of hospitals recognized that all pharmacists go to round regularly. They directly work with suffered patients and prescribers, and give services not only related to medicines, all these services provided by the clinical pharmacist for the inpatient care resulted in significantly improved care with no harm. ${ }^{24}$

Effect on Discharged Patients: Patients that were hospitalized and then discharged home show no further continuity in care, changes made in medicine administration, due to this, the side effects of drugs may show. The main objective was to visualize medicine related issues during hospitalization and after discharge and check patient counseling effect on patient and also to follow pharmacist to prevent side effects. A randomized trial is done on the 77 patients who are recently being discharged to home from medicine service provided at hospital. Pharmacist counseling continued to patients in intervention group and they follow telephone call for few days. The outcome of this effort is that adverse effect of drugs decreased up to $30 \%$ in intervention group where as $1 \%$ in other group. ${ }^{25}$

\section{Conclusion}

The review study shows that pharmacist and physician co management in order to control blood pressure is significant at decreasing blood pressure and rate of blood pressure control is improved. The pharmacist can also improve medication adherence, quality of life and patient knowledge about diseases and medication and give satisfaction to patient. In addition, 
the use of drugs is reduced. This study emphasizes that intervention of pharmacist in medication management or therapy is health effective. As a member of health care team pharmacist can increase the number of health outcomes. Hypertension is an important risk factor contributes to present epidemic cardiovascular problems with the pharmacist intervention in controlling of blood pressure its risk is going to decrease.

\section{References}

1. Bhowmik D, Das B, KumarKS.Role of community pharmacist; care of hypertensive patients. International $J$ Pharma Sci Res 2010;1(8):23.

2. Polgreen LA et al.Cost-Effectiveness of a PhysicianPharmacist Collaboration Intervention to Improve Blood Pressure ControlNovelty and Significance. Hypertension 2015;66(6):1145-51.

3. Biradar $\mathrm{S}$ et al.Importance of role of pharmacist mediated adherence in hypertensive patients a brief overview. 2012;1(1):11-5.

4. Gorman BK,Porter JR.Social networks and support, gender, and racial/ethnic disparities in hypertension among older adults. Population Res Policy Rev 2011;30(6):885-911.

5. Morgado M, Rolo S,Castelo-Branco M.Pharmacist intervention program to enhance hypertension control: a randomised controlled trial. Int J Clin Pharma 2011;33(1):132-40.

6. Khorosh M, Holovanova I, Harkavenko, M. Risk factors for development of hypertension in Poltava region 2016;69;2(cz. II):190-96.

7. Delacroix S, Chokka R,Worthley S.Hypertension: pathophysiology and treatment. J Neurol Neurophysiol 2014;5(250):doi: 10.4172/2155-9562.1000250.

8. Mungreiphy N, Kapoor S,Sinha R.Association between BMI, blood pressure, and age: study among Tangkhul Naga tribal males of Northeast India. J Anthropol 2011:doi:10.1155/2011/748147.

9. Lee JK et al. Optimizing pharmacotherapy in elderly patients: the role of pharmacists. Integrated Pharma Res Practice 2015;4:101.

10. Zheng S,James PA.The potency of team-based care interventions for hypertension. Arch Intern Med 2009;169(19):1748-55.

11. Saleem F et al, Pharmacist intervention in improving hypertension-related knowledge, treatment medication adherence and health-related quality of life: a non-clinical randomized controlled trial. Health Expectations 2015;18(5):1270-81.

12. Ramanath $\mathrm{K}$ et al, A study on impact of clinical pharmacist interventions on medication adherence and quality of life in rural hypertensive patients. $J$ Young Pharmacists 2012;4(2):95-100.

13. Santschi V, Tsuyuki RT,Paradis G.Evidence for pharmacist care in the management of hypertension. Can Pharma J/Revue des Pharmaciens du Canada 2015;148(1):13-6.

14. Borenstein JE et al.Physician-pharmacist comanagement of hypertension: a randomized, comparative trial. Pharmacotherapy: J Human Pharmacol Drug Therapy 2003;23(2):209-16.

15. Carter BL et al.A cluster randomized trial to evaluate physician/pharmacist collaboration to improve blood pressure control. J Clin Hypertension 2008;10(4):260-71.

16. Garçao JA,Cabrita J.Evaluation of a pharmaceutical care program for hypertensive patients in rural Portugal. $J \mathrm{Am}$ Pharma Assoc 2002;42(6):858-64.

17. Carter BL, BosworthHB,Green BB.The hypertension team: the role of the pharmacist, nurse, and teamwork in hypertension therapy. J Clin Hypertension 2012;14(1):5165.

18. Charrois TL et al. Improving hypertension management through pharmacist prescribing; the rural Alberta clinical trial in optimizing hypertension (Rural RxACTION): trial design and methods. Implementation Sci 2011; 6(1):94. doi: 10.1186/1748-5908-6-94.

19. Cheema E, Sutcliffe P,Singer DR. The impact of interventions by pharmacists in community pharmacies on control of hypertension: a systematic review and meta-analysis of randomized controlled trials. Br J Clin Pharmacol 2014;78(6):1238-47.

20. Njie GJ et al, Peer Reviewed: Reducing Medication Costs to Prevent Cardiovascular Disease: A Community Guide Systematic Review. Preventing chronic disease 2015;12: http://dx.doi.org/10.5888/pcd12.150242.

21. Porter ME. What is value in health care? New Engl J Med 2010;363(26):2477-81.

22. Carter BL,Elliott WJ.The role of pharmacists in the detection, management, and control of hypertension: a national call to action. Pharmacotherapy: J Human Pharmacol Drug Therapy 2000;20(2):119-22.

23. McDonald HP, Garg AX,Haynes RB. Interventions to enhance patient adherence to medication prescriptions: scientific review. JAMA 2002;288(22):2868-79.

24. Boston MDS. Clinical pharmacists and inpatient medical care. Arch Intern Med 2006;166:955-64.

25. Schnipper JL et al, Role of pharmacist counseling in preventing adverse drug events after hospitalization. Arch Internal Med 2006;166(5):565-71. 